\section{Codependency of $\mathrm{H} 2 \mathrm{~B}$ monoubiquitination and nucleosome reassembly on Chd1}

\author{
Jung-Shin Lee, ${ }^{1}$ Alexander S. Garrett, ${ }^{1}$ \\ Kuangyu Yen, ${ }^{2}$ Yoh-Hei Takahashi, ${ }^{1}$ Deqing Hu, ${ }^{1}$ \\ Jessica Jackson, ${ }^{3}$ Christopher Seidel, ${ }^{1}$ \\ B. Franklin Pugh, ${ }^{2}$ and Ali Shilatifard ${ }^{1,4}$ \\ ${ }^{1}$ Stowers Institute for Medical Research, Kansas City, Missouri \\ 64110, USA; ${ }^{2}$ Center for Eukaryotic Gene Regulation, \\ Department of Biochemistry and Molecular Biology, The \\ Pennsylvania State University, University Park, Pennsylvania \\ 16802, USA; ${ }^{3}$ Department of Biochemistry, St. Louis University \\ School of Medicine, St. Louis, Missouri 63104, USA
}

\begin{abstract}
Monoubiquitination of histone H2B on Lys 123 (H2BK123ub) is a transient histone modification considered to be essential for establishing H3K4 and H3K79 trimethylation by Set1/COMPASS and Dot1, respectively. Here, we identified Chd1 as a factor that is required for the maintenance of high levels of H2B monoubiquitination, but not for H3K4 and H3K79 trimethylation. Loss of Chd1 results in a substantial loss of H2BK123ub levels with little to no effect on the genome-wide pattern of $\mathrm{H} 3 \mathrm{~K} 4$ and $\mathrm{H} 3 \mathrm{~K} 79$ trimethylation. Our data show that nucleosomal occupancy is reduced in gene bodies in both chd1s and, as has been shown, K123A mutant backgrounds. We also demonstrated that Chd1's function in maintaining H2BK123ub levels is conserved from yeast to humans. Our study provides evidence that only small levels of $\mathrm{H} 2 \mathrm{BK} 123 \mathrm{ub}$ are necessary for full levels of $\mathrm{H} 3 \mathrm{~K} 4$ and $\mathrm{H} 3 \mathrm{~K} 79$ trimethylation in vivo and points to a possible role for $\mathrm{Chd} 1$ in positively regulating gene expression through promoting nucleosome reassembly coupled with H2B monoubiquitination.
\end{abstract}

Received January 9, 2012; revised version accepted March 26, 2012.

As fundamental components of chromatin, the histone proteins are subjected to a variety of post-translational modifications that function in diverse processes (Bhaumik et al. 2007; Smith and Shilatifard 2010). In order to understand the machinery that implements these modifications and the cellular contexts in which they function, we developed a biochemical screen called global proteomic analysis in Saccharomyces cerevisiae (GPS) (Schneider et al. 2004). This screen and other genetic screens identified numerous factors required for the proper implementation of different H3K4 and H3K79 methylation states. Notably, these include the histone H2B monoubiquitinase complex Rad6/Bre1's requirement for histone H3K4 and

[Keywords: chromatin; transcription; ubiquitination; histone] ${ }^{4}$ Corresponding author.

E-mail ash@stowers.org

Article is online at http://www.genesdev.org/cgi/doi/10.1101/gad.186841.112.
H3K79 trimethylation by Set1/COMPASS and Dot1, a process termed histone cross-talk (Dover et al. 2002; Sun and Allis 2002; Hwang et al. 2003; Wood et al. 2003a; Schneider et al. 2004; Smith and Shilatifard 2010). Other factors were identified by GPS to be required for proper H3K4 and/or H3K79 methylation, including the Paf1 complex consisting of Paf1, Rtf1, Cdc73, and Ctr9 (Krogan et al. 2003; Wood et al. 2003b); the CTD kinases Bur1/Bur2 and Ctk1/ Ctk2/Ctk3 (Wood et al. 2005, 2007); the cell cycle regulator SBF (Schulze et al. 2009); and the N-terminal acetyltransferase Ard1 (Takahashi et al. 2011).

Collectively, these studies using antibodies recognizing histone H3K4 and/or H3K79 methylation showed numerous connections between the implementation of histone $\mathrm{H} 2 \mathrm{~B}$ monoubiquitination and H3K4 methylation. In this study, we used a recently generated antibody recognizing yeast $\mathrm{H} 2 \mathrm{~B}$ monoubiquitination and identified the chromatin remodeler Chd1 as a novel factor required for the maintenance of high levels of histone $\mathrm{H} 2 \mathrm{~B}$ monoubiquitination. Importantly, the status of H3K4 and H3K79 methylation was unaffected in the chd1s background, explaining why Chd1 was not identified in the earlier GPS screens using H3K4- and H3K79-specific antibodies. Despite the earlier studies suggesting integration of $\mathrm{H} 2 \mathrm{~B}$ monoubiquitination and $\mathrm{H} 3$ methylation machineries, this study demonstrates that only low levels of monoubiquitination are required for proper levels of H3K4 and H3K79 methylation. Also, recent studies suggested other possible roles associated with histone $\mathrm{H} 2 \mathrm{~B}$ monoubiquitination, distinct from histone cross-talk (Pavri et al. 2006; Tanny et al. 2007; Fleming et al. 2008; Batta et al. 2011; Schulze et al. 2011), including effects on nucleosome positioning (Batta et al. 2011). We therefore used genome-wide expression and nucleosome occupancy analysis in a chd1s strain and a strain defective for $\mathrm{H} 2 \mathrm{~B}$ monoubiquitination and propose an evolutionarily conserved function for Chd1 in positively regulating gene expression through promoting nucleosome reassembly coupled with H2B monoubiquitination.

\section{Results and Discussion}

Loss of H2B monoubiquitination with normal levels of H3K4 and H3K79 methylation

Prior GPS screens using antibodies specific to either H3K4 or H3K79 methylation identified a requirement for numerous factors connected to $\mathrm{H} 2 \mathrm{~B}$ monoubiquitination in proper $\mathrm{H} 3$ methylation (Smith and Shilatifard 2010). Here, we instead performed a GPS-style screen with antibodies specific to histone $\mathrm{H} 2 \mathrm{~B}$ monoubiquitination and identified Chd1 as a novel factor required for maintaining proper levels of histone $\mathrm{H} 2 \mathrm{~B}$ monoubiquitination (Fig. 1A, lane 12). Although $\mathrm{H} 2 \mathrm{~B}$ monoubiquitination levels are significantly reduced, H3K4 and H3K79 methylation levels were unchanged (Fig. 1B), indicating why Chd1 was not previously identified in screens probing the methylation status of these residues. In the absence of Bre1, the E3 ligase required for $\mathrm{H} 2 \mathrm{~B}$ monoubiquitination by Rad6 (Wood et al. 2003a), we cannot detect any histone H2B monoubiquitination or any histone H3K4 and/or H3K79 trimethylation (Fig. 1B, lanes 7-10), validating the specificity of our antibodies. 


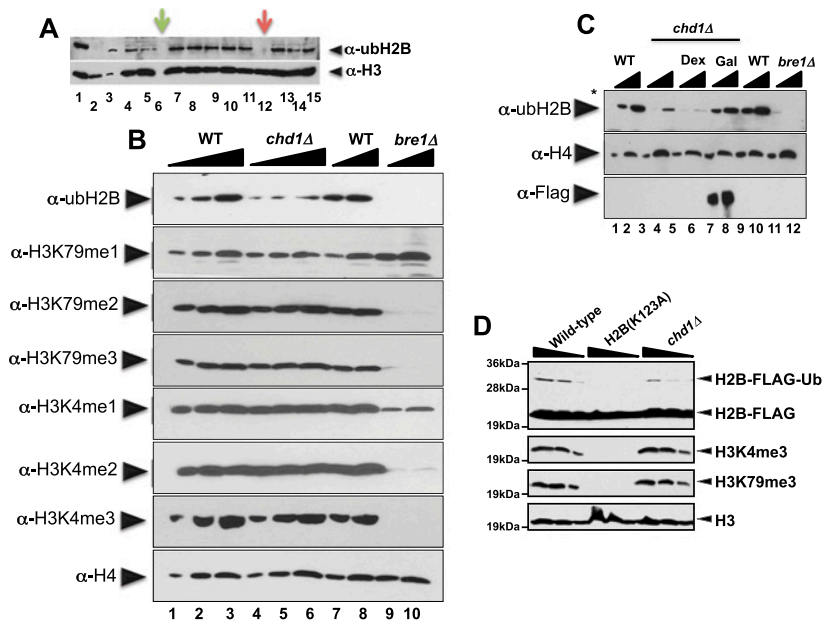

Figure 1. Chd1 is required for the maintenance of high levels of $\mathrm{H} 2 \mathrm{~B}$ monoubiquitination but not $\mathrm{H} 3 \mathrm{~K} 4$ or $\mathrm{H} 3 \mathrm{~K} 79$ trimethylation. Cell extracts from the nonessential yeast gene deletion mutants were subjected to SDS-PAGE and analyzed by Western blotting using $\mathrm{H} 2 \mathrm{~B}$ monoubiquitination-specific antibodies. Lane $\mathrm{H}$ of plate \#148 of the collection is shown in A. The red arrow indicates the position in the plate containing the chd1s strain. The green arrow points to the empty well in the collection, used as the plate marker. (B) Cell extracts from wild-type (WT) and chd1s and bre $1 \Delta$ strains were prepared and used in a titration analysis. Each extract was analyzed by its application to SDS-PAGE and tested for the presence of modified H3K4 and H3K79 mono-, di-, and trimethylation (H3K4me1-3 and H3K79me1-3) and H2B monoubiquitination (ubH2B) or total $\mathrm{H} 4$ using the appropriate antibodies. (C) A direct role for Chd1 in the regulation of histone $\mathrm{H} 2 \mathrm{~B}$ monoubiquitination was demonstrated by complementation of the chd1s strains with a vector carrying the CHD1 coding sequence under the GAL1 promoter. In the presence of galactose, Chdl is expressed (shown in lanes 7,81 , as evident by Western signal with anti-Flag, and yeast cells are restored for monoubiquitinated H2B. (D) CHD1 was also deleted in a parental background that contains a sole copy of Flagtagged histone $\mathrm{H} 2 \mathrm{~B}$ (to detect both wild-type and monoubiquitinated $\mathrm{H} 2 \mathrm{~B})$. Extracts prepared from wild-type and chd1s strains were analyzed by SDS-PAGE and Western with the indicated antibodies.

We confirmed a specific role for Chd1 in regulating histone $\mathrm{H} 2 \mathrm{~B}$ monoubiquitination levels by complementing the chd1A strain with a vector carrying Flag::CHD1 under the GAL1 promoter (Fig. 1C). In this complementation study, strains carrying the CHD1 gene under the GAL1 promoter (Fig. 1C, lanes 5-8) were grown in synthetic medium (SD and SGal) without yeast extract. However, the chd1s strain shown in Figure 1C, lanes 3 and 4, was grown in YPD. The marginal differences observed in chd1s between the H2B monoubiquitination levels in Figure 1C, lanes 3 and 4 and lanes 5 and 6, could be due to the culture conditions. We confirmed the requirement for Chd1 in $\mathrm{H} 2 \mathrm{~B}$ monoubiquitination in a chd1d strain where the sole copy of histone $\mathrm{H} 2 \mathrm{~B}$ is tagged with Flag in order to detect the endogenous copies of both $\mathrm{H} 2 \mathrm{~B}$ and monoubiquitinated H2B (Fig. 1D).

\section{Genome-wide analyses of histone H3K4 and H3K79 trimethylation under reduced $H 2 B$ monoubiquitination levels}

Since the deletion of CHD1 resulted in a substantial decrease in bulk histone $\mathrm{H} 2 \mathrm{~B}$ monoubiquitination levels (Fig. 1), we determined the genome-wide pattern of histone H3K4 and H3K79 trimethylation via chromatin immunoprecipitation (ChIP) coupled with deep sequencing (ChIP-seq) (Fig. 2A-C). Our data show that the global patterns of both $\mathrm{H} 3 \mathrm{~K} 4$ and $\mathrm{H} 3 \mathrm{~K} 79$ trimethylation were not significantly altered, although histone H2B monoubiquitination levels were decreased in these cells. An overview of the ChIP-seq signal on the entire $\sim 230-\mathrm{kb}$ chromosome I (Fig. 2A) indicates that the patterns of enrichment for the given histone marks are not grossly altered in level or intensity upon CHD1 deletion. In order to more quantitatively determine the extent of genomewide changes in these marks, we computed an occupancy enrichment metric as the ratio of the sum of normalized read levels of each histone mark in the region of \pm 500 base pairs (bp) surrounding each gene start site divided by the normalized read sum of the associated whole-cell extract control sample (input) in the same region. We then compared the occupancy enrichment ratios between the wild-type and chd1د strains for both marks (Fig. 2B,C) and found that the Pearson correlation coefficients for H3K4 and H3K79 trimethylation were 0.928 and 0.787 , respectively. When we assessed only the normalized read sums surrounding gene start sites (without dividing by the whole-cell extract sample), both marks had a correlation coefficient $>0.95$ between the wild-type and chd1s strains.

\section{Chd1, histone H2B monoubiquitination, nucleosomal occupancy, and gene expression}

To determine the effect of the loss of Chd1 on overall gene expression in yeast cells, we performed RNA sequencing (RNA-seq) analyses in wild-type and chd1s strains (Fig. 2D-G). We first checked the expression of the CHD1 locus in the chd1s strain and confirmed that the deletion resulted in complete loss of CHD1 expression (Fig. 2D). Using an adjusted $P$-value threshold of 0.05 , we found that 31 genes were down-regulated and 118 genes were up-regulated after CHD1 deletion (Fig. 2E). A visual example of loss of expression for some highly expressed genes in the wild-type strain-namely, HOM3 (Fig. 2F) and ARN2-are shown (Fig. 2G). Our RNA-seq studies and a recent microarray study on the gene expression regulatory and nucleosome organization function of Chd1 (Gkikopoulos et al. 2011) demonstrate that the expression of very few genes are altered in the absence of Chd1.

Monoubiquitination of histone $\mathrm{H} 2 \mathrm{~B}$ plays an important positive role in transcription by promoting nucleosome reassembly in the wake of a transcribing RNA polymerase, based on the observation that nucleosomes weakly reassemble at highly active genes in an H2BK123A or rad64 mutant (Batta et al. 2011). Since Chd1 is required for bulk histone H2B monoubiquitination levels (Fig. 1), we asked whether Chdl could also function in the reassembly process as well. This led us to test whether chd1s strains are defective for nucleosome assembly and whether such a defect predominates at genes that are positively regulated by $\mathrm{H} 2 \mathrm{~B}$ monoubiquitination or by Chd1. Our studies show that gene regulation by $\mathrm{H} 2 \mathrm{~B}$ monoubiquitination and Chd 1 is modestly correlated, reflecting the minimal impact of the chd1s mutant on gene expression (data not shown). In our studies, nucleosomes were mapped by MNase ChIP-seq using H3 antibodies in wild-type and isogenic mutant strains, as described by Batta et al. (2011).

In Figure 3A, genic nucleosomal arrays were aligned by their midpoints and sorted by array length, with heat map colors reflecting nucleosome occupancy /or changes in 
Lee et al.

A

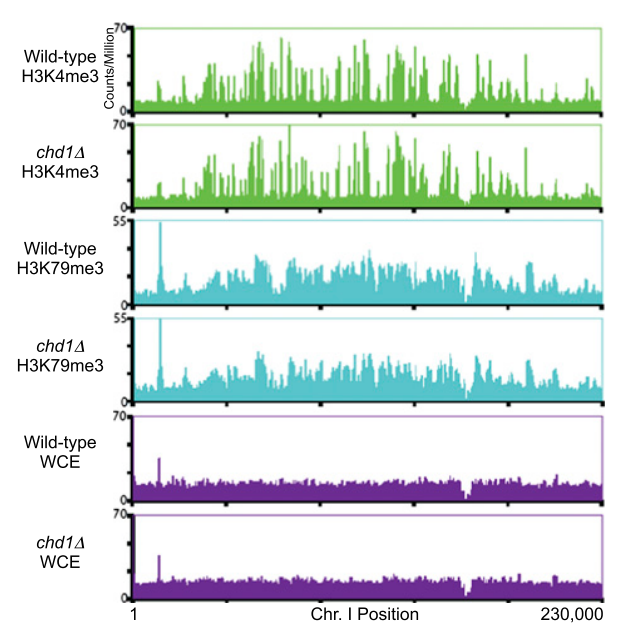

$\mathrm{F}$

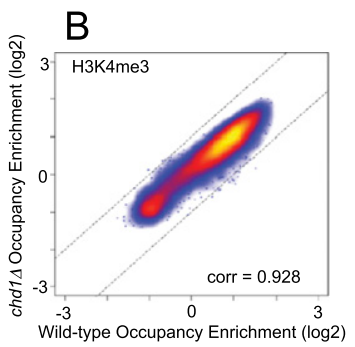

C

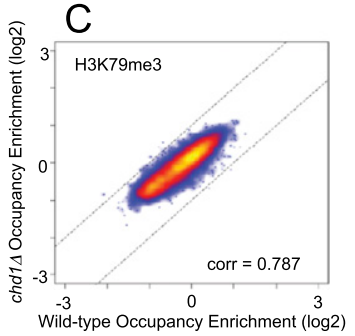

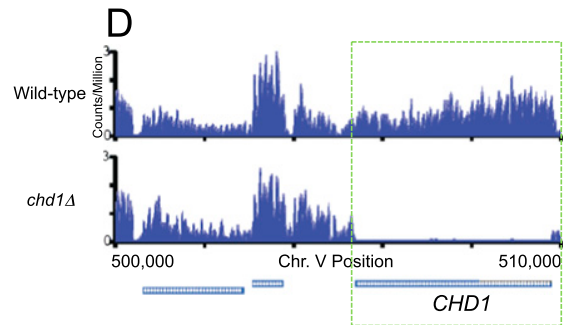

$E$

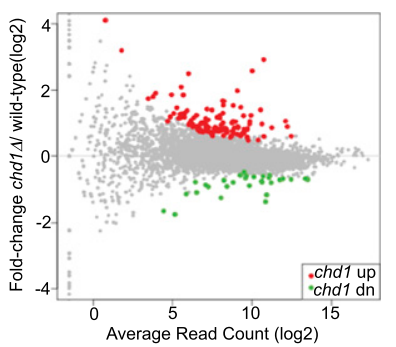

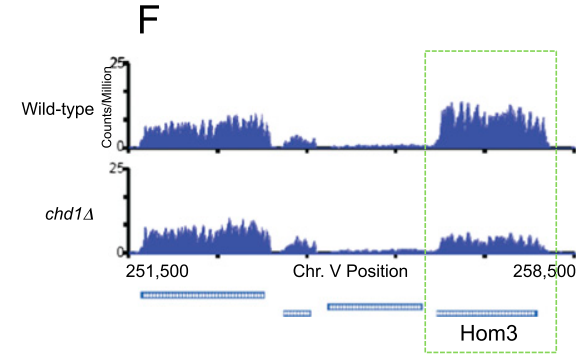

G

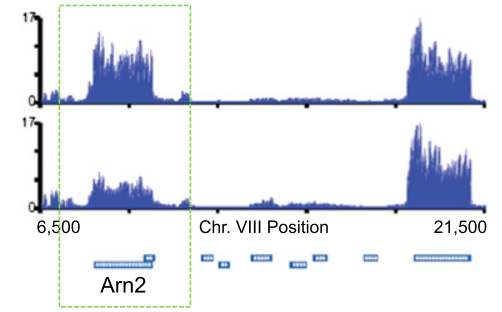

Figure 2. Genome-wide occupancy of histone H3K4 and H3K79 methylation and gene expression pattern as a result of the loss of CHD1. Global occupancy for H3K4 and H3K79 trimethylation in the presence and absence of Chd1 were determined using ChIP-seq in yeast cells. ChIPs with antibodies specific to trimethylated H3K4 and H3K79 were performed and the resulting DNA sequenced. $(A)$ A track file for chromosome I of yeast. $(B, C)$ Scatter plots depict the wild-type versus chd1s occupancy enrichment for the immunoprecipitation indicated in the top left. Normalized read sums of the immunoprecipitation divided by the normalized read sums of the whole-cell extract control within a region $\pm 500 \mathrm{bp}$ of each gene start site is plotted for the wild-type and mutant strain. The correlation coefficient shown in the bottom right is the Pearson correlation computed about the $\log 2$ transformed occupancy enrichment. The dotted lines indicate positions of a twofold change in occupancy. Scatter plot point density is indicated by color. (Blue) Low; (yellow) high. (D) RNA-seq track diagram showing complete loss of $C H D 1$ transcript in the deletion strain. (E) MA plot depicting genes differentially expressed at a false discovery rate (FDR) $<0.05$ as a result of loss of $C H D 1$. $(F, G)$ RNA-seq track diagrams of two genes, HOM3 $(F)$ and $A R N 2(G)$, that are down-regulated in the absence of CHD1.

occupancy) (right panel). Defects in occupancy are evident by "cooler" colors in the chd1s mutant compared with wild type. We found that shorter genes were largely unaffected in the chd1s strain and that longer genes show diminished occupancy within the $5^{\prime}$ region of gene bodies. Analyses of global nucleosome organization in wild-type, a K123A strain, and a chd1s strain indicate that in the absence of H2B monoubiquitination or Chd1, genes that are positively regulated by histone $\mathrm{H} 2 \mathrm{~B}$ monoubiquitination or Chd1 (i.e., down-regulated in expression in a K123A or chd1s mutant) fail to adequately assemble nucleosomes $+2,+3$, and +4 (Fig. 3B). The +1 nucleosome position was generally unaffected, and those further downstream from +4 were generally less affected (but not unaffected). Indeed, Chd 1 and $\mathrm{H} 2 \mathrm{~B}$ monoubiquitination had differing contributions toward the $3^{\prime}$ ends of genes.

Genes that are negatively regulated by $\mathrm{H} 2 \mathrm{~B}$ monoubiquitination or Chdl (up-regulated in the mutants) (Fig. 3C) did not have their nucleosome occupancy levels substantially impacted by the loss of these factors. Interestingly, genes that are negatively regulated by $\mathrm{H} 2 \mathrm{~B}$ monoubiquitination generally lacked a -1 nucleosome in a K123A mutant. This is consistent with the prior report that H2Bub stabilizes the -1 nucleosome at these genes (Batta et al. 2011). Its loss results in elevated transcription, but also a now-manifested defect in nucleosome assembly in the body of the genes, and this may have dampened the observed elevated level of transcription. Thus, H2Bub and Chd 1 may have differing roles at the promoters, being functionally unlinked there, as compared with the genic regions.

\section{Chd1 is required for the maintenance of high levels} of histone H2B monoubiquitination in human cells

Although it was demonstrated that the human Chdl is capable of interacting with H3K4 methylated histones, the yeast enzyme lacks this ability, suggesting that yeast and human Chdl differed in some aspects of their biological function (Sims et al. 2005, 2007). Therefore, we wanted to determine whether human Chd1 is required for the maintenance of high levels of $\mathrm{H} 2 \mathrm{~B}$ monoubiquitination, similar to our findings in yeast. The human genome encodes for at least nine homologs to yeast Chd1, with hChd1 and hChd2 being most related to yeast Chd1 (Sims and Wade 2011). We used a shRNA to target the closely 

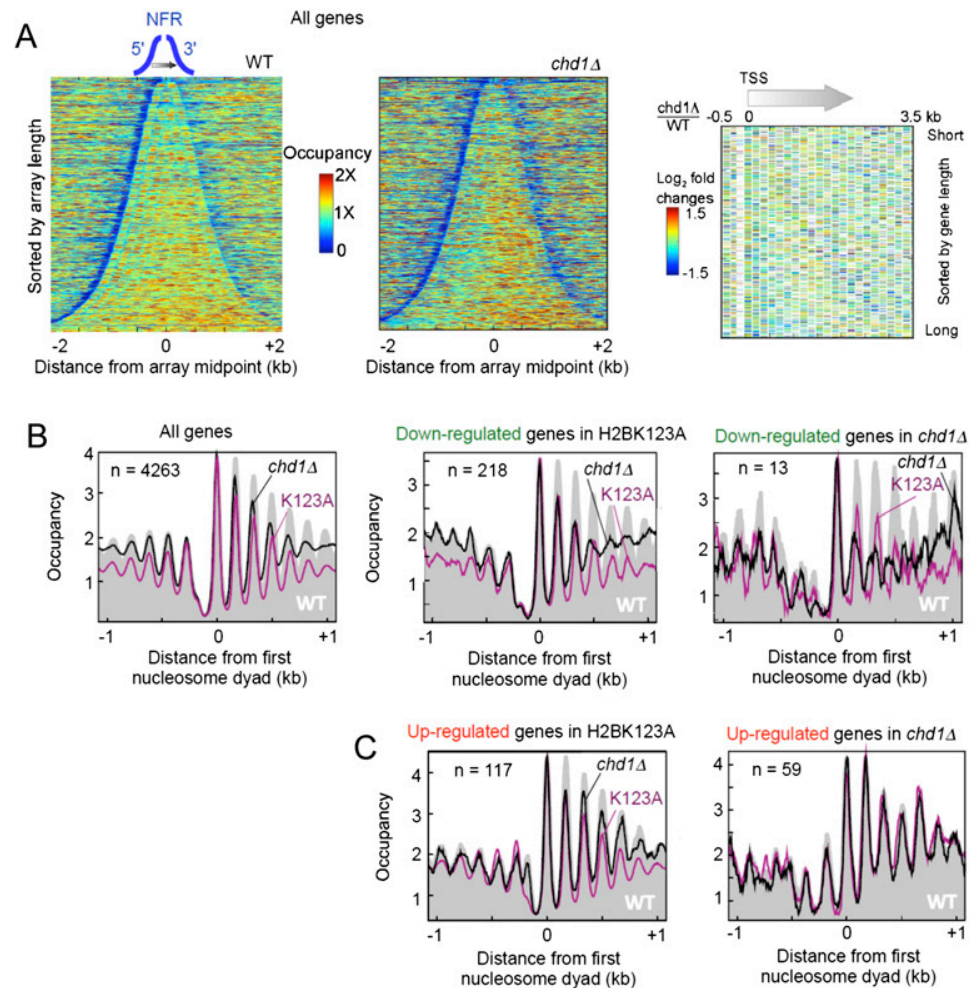

Figure 3. Nucleosomal occupancy and gene expression as a result of the loss of Chd1. (A) Heat maps of nucleosomal occupancies in wild-type (WT) and chd1s strains. Each row represents a genic nucleosomal array; arrays are sorted by array length and aligned by array midpoint (illustrated above the plot). Blue areas are the $5^{\prime}$ and 3' nucleosome-free regions (NFRs). Color bar represents the nucleosomal occupancy from zero (dark blue) to genomic average (green) to twice the genomic average (dark red). Occupancy was measured by extending the dyad position of each nucleosomal tag $73 \mathrm{bp}$ in both directions, then summing all tag coverage at each base pair. A difference map is shown in the right panel. Details of plot generation can be found in Batta et al. (2011). $(B, C)$ Composite distribution of nucleosomal tags in wild type (filled gray), H2BK123A, and the chd1s mutant, aligned by the wild-type consensus position of the first nucleosome dyad for the indicated subsets of genes. Individual tag positions were defined by a single coordinate representing the nucleosome dyad. The subset of genes that are either up-regulated or down-regulated in the K123A or chd1s mutant is shown.
+1 nucleosome occupancy. These other factors may include components of the TFIID and SWR1 complexes, which have been shown to bind to the +1 nucleosome (Koerber et al. 2009; Rhee and Pugh 2012). Interestingly, RNA polymerase II appears to be sensing the status of nucleosomes downstream from its position, perhaps as a checkpoint for initiation and/or early elongation. Our results also suggest that proper nucleosome organization in the body of the genes is a positive effector of transcription.

It is not clear to us at this time how mechanistically Chd1 exerts its H2B monoubiquitination regulatory activity. Given Chd1's role in chromatin remodeling, one can envision a model by which Chd1 remodels nucleosomes in a way that allows access to Rad6/Bre1, therefore resulting in monoubiquitination of histone $\mathrm{H} 2 \mathrm{~B}$ on nucleosomes. An alternative hypothesis is that monoubiquitinated $\mathrm{H} 2 \mathrm{~B}$ is removed following $\mathrm{H} 3 \mathrm{~K} 4$ and $\mathrm{H} 3 \mathrm{~K} 79$ methylation and that Chd1's presence is inhibitory to the deubiquitination activity of Ubp8/Ubp10. In the absence of Chd1, Ubp8/ Ubp10 functions without regulation. Further studies in this regard should provide insight into the possible existence of functional antagonism between Ubp8/Ubp10 and Chd1.

In conclusion, the discovery of the evolutionarily conserved role for Chd 1 in the maintenance of histone H2B monoubiquitination levels, yet not that of $\mathrm{H} 3 \mathrm{~K} 4$ and $\mathrm{H} 3 \mathrm{~K} 79$ trimethylation, provides us with new insight into the complex network of factors functioning in the regulation of chromatin modifications, nucleosomal occupancy/positioning, and the regulation of gene expression. These findings in yeast are highly relevant in metazoans, as a recent study reported low levels of H2B monoubiquitination, but normal H3K4 trimethylation levels, during myogenic differentiation (Vethantham et al. 2012). Understanding the mechanism of Chd1's regulation of $\mathrm{H} 2 \mathrm{~B}$ monoubiquitination will require much further analyses in both yeast and mammalian systems. related human homolog of yeast Chd1 (NM_001270), reducing hChd 1 levels by $>80 \%$ in 293 cells (Fig. 4, lanes 4-6). Similar to yeast Chd1, reduced hChd1 levels result in reduction of $\mathrm{H} 2 \mathrm{~B}$ monoubiquitination levels, with little to no effect on H3K4 and H3K79 trimethylation levels. As a control, we confirmed that reducing human Brel levels (Bre1A in this case) results in substantial loss of both H2B monoubiquitination and H3K4 and H3K79 trimethylation levels (Fig. 4, lanes 7-9).

Our studies provide a working model of how Chd1 and/ or histone $\mathrm{H} 2 \mathrm{~B}$ monoubiquitination regulate transcription. We envision that the combined action of nucleosome stabilization by $\mathrm{H} 2 \mathrm{~B}$ monoubiquitination and nucleosome reassembly by Chd1 promotes proper nucleosome organization in the wake of elongating RNA polymerase II. RNA polymerase II appears to be unable to move from the promoter position when nucleosomes fail to properly assemble in the body of genes (Batta et al. 2011). What is surprising is that the +1 nucleosome is utterly unaffected by defects in reassembly by Chd1, which suggests that there are other factors that control

\section{Materials and methods}

Yeast strains and immunoblot analyses

Cell extracts were prepared from exponentially growing yeast cells (at $\left.\sim 1.0 \mathrm{OD}_{600 \mathrm{~nm}}\right)$ in YPD ( $1 \%$ yeast extract, $2 \%$ peptone, $2 \%$ dextrose $)$ and analyzed by Western blotting. For CHD1 complementation, cells containing the pRS315 vector bearing Flag-tagged CHD1 under the control of the GAL1 promoter were cultured in SD medium deficient of leucine overnight at $30^{\circ} \mathrm{C}$; transferred to synthetic medium containing dextrose or galactose, respectively; and incubated for $6 \mathrm{~h}$ at $30^{\circ} \mathrm{C}$. Cell extracts were prepared and analyzed by Western blotting with antibodies generated by our laboratory, except for anti-H3K79 trimethylation (Abcam, ab2621) and anti-Flag (Sigma, F3165).

\section{ChIP-seq and RNA-seq for the chd $1 \Delta$ strain}

ChIPs were performed with cell lysates prepared from exponentially growing wild-type and chd1s strains to $\mathrm{OD}_{600} \mathrm{~nm}$ of 1.0 according to previously described methods (Wood et al. 2005). Ten nanograms of DNA prepared from each ChIP was amplified using LM-PCR and subjected to 50-nucleotide (nt) single-read sequencing on a HiSeq2000 instrument. Reads were normalized to total tag count (reads per million). In our studies, 


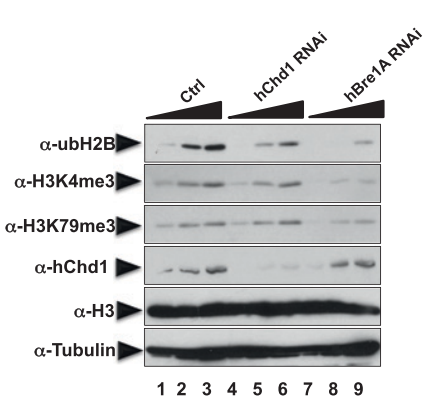

Figure 4. A conserved role of $\mathrm{Chd} 1$ in the maintenance of $\mathrm{H} 2 \mathrm{~B}$ monoubiquitination levels from yeast to humans. Human 293 cells were transfected with control, hChdl, and hBrelA shRNA as indicated. Following $96 \mathrm{~h}$ of incubation, the RNAi efficiency for Chd1 and Bre 1 and their effect on the patterns of H2B monoubiquitination and H3K4 and H3K79 methylation were tested by Western analyses using the appropriate antibodies. Histone $\mathrm{H} 3$ and Tubulin were used as load controls.

we made the assumption that the bulk $\mathrm{H} 3$ levels remain equal, similar to data shown in Figure 1. ChIP-seq alignments were processed by Bowtie version 0.12.7 (Langmead et al. 2009), allowing up to two mismatches and suppressing alignments if $>10$ existed.

For RNA-seq, total RNAs were prepared from the exponentially growing wild-type and chd1 $1 \Delta$ strains to $\sim 0.8 \mathrm{OD}_{600} \mathrm{~nm}$ using the hot phenol method. Harvested cells were broken with $65^{\circ} \mathrm{C}$ acidic phenol, and the lysates were extracted by phenol/chloroform/isoamylalcohol three times. Total RNAs were prepared from these lysates by ethanol precipitation. The library was prepared using the Tru-seq mRNA kit (catalog no. FC-122-1001) from Illumina. RNA-seq alignments were processed with TopHat version 1.3.1 (Trapnell et al. 2009). DESeq version 1.4.1 (Anders and Huber 2010) was used to assess differential expression at the adjusted $P$-value threshold $<0.05$. Ensembl 63 annotations were used for transcription start site and expression analysis. Sequencing data were deposited at Gene Expression Omnibus accession number GSE36334.

\section{Nucleosome mapping}

Nucleosome positions were mapped in a chd1s strain as described by Batta et al. (2011). In brief, cells were subjected to formaldehyde crosslinking, harvested, then used to prepare chromatin pellets. Pellets were digested with MNase to $\sim 80 \%$ mononucleosomes, as detected by gel electrophoresis. Nucleosomes were immunopurified with $\mathrm{H} 3$ antibodies under stringent conditions that were dependent on prior cross-linking to retain nucleosomal DNA. Eluted DNA was gel-purified in the mononucleosomal size range and used to construct Illumina sequencing libraries. After mapping to the yeast genome, total tag counts were normalized to be equal between wild-type, chd1s, and K123A strains. Data for the latter were obtained from Batta et al. 2011. This normalization therefore sets the total genome-wide nucleosome occupancy to be the same among the different strains, whether or not they are, in fact, the same. This differs from the normalization used by Batta et al. (2011), which sets the relative total tag count among samples to be equivalent to the relative chromosomal histone content measured by immunoblotting of histones. Consequently, bulk pattern intensities may differ. Since conclusions drawn here are based on pattern changes relative to nearby nucleosomes, these differences in normalization do not affect the conclusions. Individual tag locations were defined by a single coordinate located at a fixed distance, D, downstream from the mapped 5' end. This represented the nucleosome dyad. The value of $\mathrm{D}$ was determined to be the median midpoint distance between all nucleosomal peak pairs in a data set. Peaks represent clusters of tags. Peak locations were determined by GeneTrack software (Albert et al. 2008). The value of $D$ was 71,68 , and 78 bp for wildtype and chd1s strains and K123A data sets, respectively.

\section{RNAi studies in mammalian cells}

293 cells were transfected with control, hChd1, and hBrelA shRNA/Open Biosystems) using Lipofectamine 2000 (Invitrogen) according to the manufacturers' instructions. After $96 \mathrm{~h}$, whole cellular lysates were extracted, and immunoblots were probed with H3K4 trimethylation, H3K79 trimethylation, and $\mathrm{H} 3$ antibodies (all generated in our laboratory) and anti-H2B monoubiquitination (Medimabs, MM-029).

\section{Acknowledgments}

We thank Dr. Edwin Smith for critical reading of the manuscript and helpful comments and suggestions, and Laura Shilatifard for editorial assistance. This study was supported by National Institutes of Health grants R01HG004160 to B.F.P., and R01CA089455 and R01GM069905 to A.S.

\section{References}

Albert I, Wachi S, Jiang C, Pugh BF. 2008. GeneTrack-a genomic data processing and visualization framework. Bioinformatics 24: 13051306.

Anders S, Huber W. 2010. Differential expression analysis for sequence count data. Genome Biol 11: R106. doi: 10.1186/gb-2010-11-10-r106.

Batta K, Zhang Z, Yen K, Goffman DB, Pugh BF. 2011. Genome-wide function of $\mathrm{H} 2 \mathrm{~B}$ ubiquitylation in promoter and genic regions. Genes Dev 25: 2254-2265.

Bhaumik SR, Smith E, Shilatifard A. 2007. Covalent modifications of histones during development and disease pathogenesis. Nat Struct Mol Biol 14: 1008-1016.

Dover J, Schneider J, Tawiah-Boateng MA, Wood A, Dean K, Johnston M, Shilatifard A. 2002. Methylation of histone H3 by COMPASS requires ubiquitination of histone H2B by Rad6. J Biol Chem 277: 28368-28371.

Fleming AB, Kao CF, Hillyer C, Pikaart M, Osley MA. 2008. H2B ubiquitylation plays a role in nucleosome dynamics during transcription elongation. Mol Cell 31: 57-66.

Gkikopoulos T, Schofield P, Singh V, Pinskaya M, Mellor J, Smolle M, Workman JL, Barton GJ, Owen-Hughes T. 2011. A role for Snf2related nucleosome-spacing enzymes in genome-wide nucleosome organization. Science 333: 1758-1760.

Hwang WW, Venkatasubrahmanyam S, Ianculescu AG, Tong A, Boone C, Madhani HD. 2003. A conserved RING finger protein required for histone H2B monoubiquitination and cell size control. Mol Cell 11: 261-266.

Koerber RT, Rhee HS, Jiang C, Pugh BF. 2009. Interaction of transcriptional regulators with specific nucleosomes across the Saccharomyces genome. Mol Cell 35: 889-902.

Krogan NJ, Dover J, Wood A, Schneider J, Heidt J, Boateng MA, Dean K, Ryan OW, Golshani A, Johnston M, et al. 2003. The Paf1 complex is required for histone $\mathrm{H} 3$ methylation by COMPASS and Dotlp: Linking transcriptional elongation to histone methylation. Mol Cell 11: 721-729.

Langmead B, Trapnell C, Pop M, Salzberg SL. 2009. Ultrafast and memory-efficient alignment of short DNA sequences to the human genome. Genome Biol 10: R25. doi: 10.1186/gb-2009-10-3-r25.

Pavri R, Zhu B, Li G, Trojer P, Mandal S, Shilatifard A, Reinberg D. 2006. Histone $\mathrm{H} 2 \mathrm{~B}$ monoubiquitination functions cooperatively with FACT to regulate elongation by RNA polymerase II. Cell 125: 703-717.

Rhee HS, Pugh BF. 2012. Genome-wide structure and organization of eukaryotic pre-initiation complexes. Nature 483: 295-301.

Schneider J, Dover J, Johnston M, Shilatifard A. 2004. Global proteomic analysis of S. cerevisiae (GPS) to identify proteins required for histone modifications. Methods Enzymol 377: 227-234.

Schulze JM, Jackson J, Nakanishi S, Gardner JM, Hentrich T, Haug J, Johnston M, Jaspersen SL, Kobor MS, Shilatifard A. 2009. Linking cell cycle to histone modifications: SBF and $\mathrm{H} 2 \mathrm{~B}$ monoubiquitination machinery and cell-cycle regulation of H3K79 dimethylation. Mol Cell 35: 626-641.

Schulze JM, Hentrich T, Nakanishi S, Gupta A, Emberly E, Shilatifard A, Kobor MS. 2011. Splitting the task: Ubp8 and Ubp10 deubiquitinate different cellular pools of H2BK123. Genes Dev 25: 2242-2247.

Sims JK, Wade PA. 2011. SnapShot: Chromatin remodeling: CHD. Cell 144: 626-626.e1. doi: 10.1016/j.cell.2011.02.019.

Sims RJ III, Chen CF, Santos-Rosa H, Kouzarides T, Patel SS, Reinberg D. 2005. Human but not yeast CHD1 binds directly and selectively to histone $\mathrm{H} 3$ methylated at lysine 4 via its tandem chromodomains. J Biol Chem 280: 41789-41792. 
Sims RJ III, Millhouse S, Chen CF, Lewis BA, Erdjument-Bromage H, Tempst P, Manley JL, Reinberg D. 2007. Recognition of trimethylated histone $\mathrm{H} 3$ lysine 4 facilitates the recruitment of transcription postinitiation factors and pre-mRNA splicing. Mol Cell 28: 665-676.

Smith E, Shilatifard A. 2010. The chromatin signaling pathway: Diverse mechanisms of recruitment of histone-modifying enzymes and varied biological outcomes. Mol Cell 40: 689-701.

Sun ZW, Allis CD. 2002. Ubiquitination of histone H2B regulates H3 methylation and gene silencing in yeast. Nature 418: 104-108.

Takahashi YH, Schulze JM, Jackson J, Hentrich T, Seidel C, Jaspersen SL, Kobor MS, Shilatifard A. 2011. Dotl and histone H3K79 methylation in natural telomeric and HM silencing. Mol Cell 42: 118-126.

Tanny JC, Erdjument-Bromage H, Tempst P, Allis CD. 2007. Ubiquitylation of histone $\mathrm{H} 2 \mathrm{~B}$ controls RNA polymerase II transcription elongation independently of histone $\mathrm{H} 3$ methylation. Genes Dev 21: 835-847.

Trapnell C, Pachter L, Salzberg SL. 2009. TopHat: Discovering splice junctions with RNA-seq. Bioinformatics 25: 1105-1111.

Vethantham V, Yang Y, Bowman C, Asp P, Lee JH, Skalnik DG, Dynlacht BD. 2012. Dynamic loss of H2B ubiquitylation without corresponding changes in H3K4 trimethylation during myogenic differentiation. Mol Cell Biol 32: 1044-1055.

Wood A, Krogan NJ, Dover J, Schneider J, Heidt J, Boateng MA, Dean K, Golshani A, Zhang Y, Greenblatt JF, et al. 2003a. Bre1, an E3 ubiquitin ligase required for recruitment and substrate selection of Rad6 at a promoter. Mol Cell 11: 267-274.

Wood A, Schneider J, Dover J, Johnston M, Shilatifard A. 2003b. The Paf1 complex is essential for histone monoubiquitination by the Rad6Brel complex, which signals for histone methylation by COMPASS and Dotlp. J Biol Chem 278: 34739-34742.

Wood A, Schneider J, Dover J, Johnston M, Shilatifard A. 2005. The Bur1/ Bur2 complex is required for histone H2B monoubiquitination by Rad6/ Brel and histone methylation by COMPASS. Mol Cell 20: 589-599.

Wood A, Shukla A, Schneider J, Lee JS, Stanton JD, Dzuiba T, Swanson SK, Florens L, Washburn MP, Wyrick J, et al. 2007. Ctk complexmediated regulation of histone methylation by COMPASS. Mol Cell Biol 27: 709-720. 


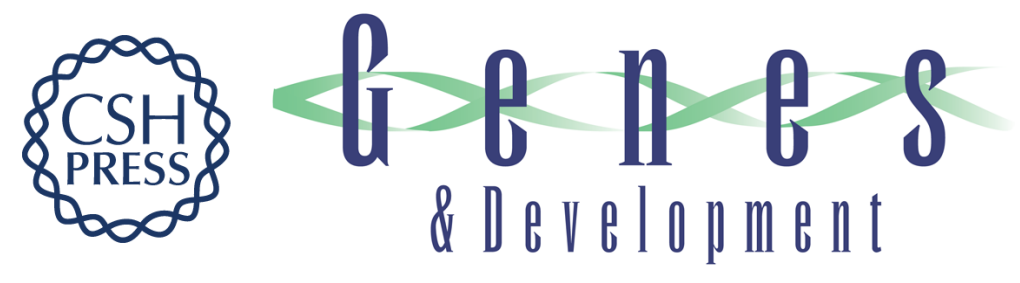

\section{Codependency of H2B monoubiquitination and nucleosome reassembly on Chd1}

Jung-Shin Lee, Alexander S. Garrett, Kuangyu Yen, et al.

Genes Dev. 2012, 26:

Access the most recent version at doi:10.1101/gad.186841.112

References This article cites 29 articles, 9 of which can be accessed free at: http://genesdev.cshlp.org/content/26/9/914.full.htmI\#ref-list-1

License

Email Alerting Receive free email alerts when new articles cite this article - sign up in the box at the top Service right corner of the article or click here.

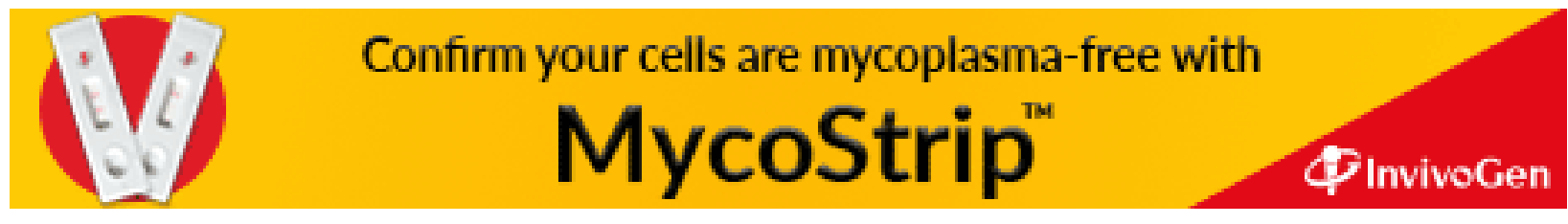

